\title{
Planning and Profit Sharing in Overlay WiFi and LTE Systems toward 5G Networks
}

\author{
Danielle Saliba ${ }^{1}$, Rodrigue Imad ${ }^{2}$, Sebastien Houcke³ ${ }^{3}$, Bachar El Hassan ${ }^{4}$ \\ ${ }^{1}$ Signal and Communication, Mines Telecom Atlantique, Brest, France \\ ${ }^{2}$ Mechatronics, University of Balamand, Al Kurah, Lebanon \\ ${ }^{3}$ Signal and Communication, Mines Telecom Atlantique, Brest, France \\ ${ }^{4}$ Telecommunication and Networking, Lebanese University, Tripoli, Lebanon \\ Email: danielle.saliba@imt-atlantique.fr, rodrigue.imad@balamand.edu.lb, sebastien.houcke@imt-atlantique.fr, \\ bachar_elhassan@ul.edu.lb
}

How to cite this paper: Saliba, D., Imad, R., Houcke, S. and El Hassan, B. (2019) Planning and Profit Sharing in Overlay WiFi and LTE Systems toward 5G Networks. Journal of Software Engineering and Applications, 12, 491-508.

https://doi.org/10.4236/jsea.2019.1211030

Received: October 29, 2019

Accepted: November 26, 2019

Published: November 29, 2019

Copyright $\odot 2019$ by author(s) and Scientific Research Publishing Inc. This work is licensed under the Creative Commons Attribution International License (CC BY 4.0).

http://creativecommons.org/licenses/by/4.0/

(c) (i) Open Access

\begin{abstract}
With the increasing demand for data traffic and with the massive foreseen deployment of the Internet of Things (IoT), higher data rates and capacity are required in mobile networks. While Heterogeneous Networks (HetNets) are under study toward 5G technology, Wireless Fidelity (WiFi) Access Points (APs) are considered a potential layer within those multiple Radio Access Technologies (RATs). For this purpose, we have proposed in this paper a novel WiFi dimensioning method, to offload data traffic from Long Term Evolution (LTE) to WiFi, by transferring the LTE energy consuming heavy users, to the WiFi network. First, we have calculated the remaining available capacity of the WiFi network based on the estimated load of each WiFi physical channel using the overlapping characteristic of the channels. Then, we were able through this dimensioning method, to calculate the minimum needed number of WiFi APs that ensure the same or better throughput for the LTE transferred users. By this method, we have ensured additional capacity in the LTE network with minimum investment cost in the WiFi network. Finally, we have estimated the profit sharing between LTE and WiFi by considering data bundles subscription revenues and the infrastructure capital and operational costs. We have calculated for each network the profit share using a coalition game theory Shapley value that pinpoints the benefit of the cooperation using the proposed dimensioning method.
\end{abstract}

\section{Keywords}

LTE WiFi Offload and Coexistence, 5G, Heterogeneous Networks, Shapley Value Profit Sharing 


\section{Introduction}

With the increasing demand for wireless communication technologies and data traffic, the main limitation in mobile networks is the lack of available licensed spectrum. Operators have limited and expensive spectrum, so they need to plan the effective utilization of their radio resources. This can be done by offloading mobile data between licensed and unlicensed spectrum [1].

Multi Radio Access Technology (RAT) solution, as the integration between Long Term Evolution (LTE) and Wireless Fidelity (WiFi), is an alleviating solution to ensure additional capacity and distribute the connected users efficiently between the Heterogeneous Networks (HetNets). WiFi is a potential candidate to support LTE in HetNets for many reasons: many studies have shown that more than $80 \%$ of mobile traffic came from indoor locations or mobile users with fixed positions [2]; WiFi Access Points (APs) are easily and quickly deployed in many residential areas and indoor environments, with affordable cost of investment and without any restrictions in hardware size or physical customization; and, most of the smart devices are equipped with WiFi capabilities. Finally, in contrast with the licensed spectrum used in LTE cellular networks, unlicensed spectrum of WiFi systems is less expensive, where 802.11x WiFi network may have better throughput and consume less power than the cellular network [3].

However, most of current WiFi networks consist of randomly deployed WiFi cells since there are no limitations or policies on WiFi AP deployment [4]. The unplanned installation of APs may cause the WiFi networks to be implemented inefficiently.

There have been several studies on WiFi APs deployment problems. In [4], the minimum required number of WiFi APs was investigated based on the active users' density, the coverage of the WiFi AP and the transmission probability of a user, without taking into consideration the WiFi network available capacity. In [5], the authors propose WiFi deployment algorithms based on realistic mobility characteristics of users to deploy WiFi APs for continuous service for mobile users, based on maximum continuous coverage where WiFi network capacity was not considered. In [6], the number of APs required for WiFi offloading with different quality of service for data delivery was quantified; however, authors just provided a feasibility study on such offloading solution through real mobility traces and did not perform any mathematical analysis for this problem.

In addition, many studies have analyzed the cooperation and offload between LTE and WiFi based on different criteria and assumptions. In [1], authors proposed a Low Amplitude Stream Injection (LASI) method to enable the simultaneous transmissions of WiFi and LTE frames in the same channel and recover the data from the conflicts. In [2], the offload to WiFi was analyzed based on the Remaining Throughput Scheme (RTS) for Wi-Fi selection. In [3], the offload to WiFi networks was proposed based on Software Defined Network (SDN) architecture to ensure WiFi Device-to-Device (D2D) link. In [7], it was proposed to transfer WiFi users to the LTE system according to the availability of Channel State Information (CSI): the random transfer, the distance-based transfer, and 
the CSI-based transfer. In [8], the offload was analyzed based on the energy cost incurred to the cellular base stations and according to a routing policy within the overlay network.

Obviously, when extra APs are installed, a WiFi network will achieve higher throughput through additional available capacity to offload LTE. Nevertheless, increasing the number of APs without any constraint is not a good solution. Therefore, it is important to investigate the minimum needed number of those APs that achieves a certain level of performance.

This paper proposes a novel method of dimensioning the WiFi network to offload the LTE. This method calculates the minimum needed number of WiFi APs based on the estimated average available capacity of the WiFi overlapped physical channels, thus the available capacity of the WiFi network. The existing WiFi network constituted of minimum one AP, will be handling the LTE offloaded traffic on top of its initial traffic, and then any needed extra capacity will be reflected by incrementing the number of WiFi APs. The proposed solution will alleviate the LTE cell energy consumption for certain calculated and defined heavy users, and thus instead of increasing the capacity and number of Base Stations (BSs) in the LTE network, we are proposing to increase the number of WiFi APs. This architecture can provide a low-cost solution compared to other solutions such as increasing the number of LTE BSs or small cells that necessitate additional cost of investment. In this case, the investment in the related Capital and Operational expenditures (CAPEX and OPEX) will be reduced; to note that the profit sharing for both networks is measured at the end of the paper by applying the gaming theory of Shapley value, to pinpoint the benefit of the coexistence and cooperation between both systems.

This paper is organized as follows: In Section 2, the overlay network model is described. In Section 3, the problem formulation along with users transfer schemes are described. The available capacity of the WiFi network and dimensioning of the needed number of WiFi APs are calculated in Section 4. Section 5 describes the profit for each network based on the gaming theory Shapley value. Section 6 shows the simulation results and the performance of the proposed solution, and Section 7 concludes the paper and introduces future works.

\section{Overlay LTE/WiFi Network Model}

We consider in this paper a network where an LTE Advanced (LTE-A) cell that operates in the licensed spectrum, also known as eNB, is covered by $K \mathrm{WiFi}$ APs ( $K$ unknown variable to be calculated) that operates in the unlicensed spectrum and that will support the transfer of heavy users from LTE-A to a WiFi with a sufficient capacity and proper available coverage.

The proposed architecture of the overlay network is depicted in Figure 1 where the eNB serves a set of Mobile Users (MUs) (or User Equipments (UEs)) that also have WiFi interfaces.

We consider that the MUs are in range with at least one or more WiFi APs. The amount of data to be downloaded or uploaded from/to the internet differs between different users, as well as for their channel conditions with the BS. 


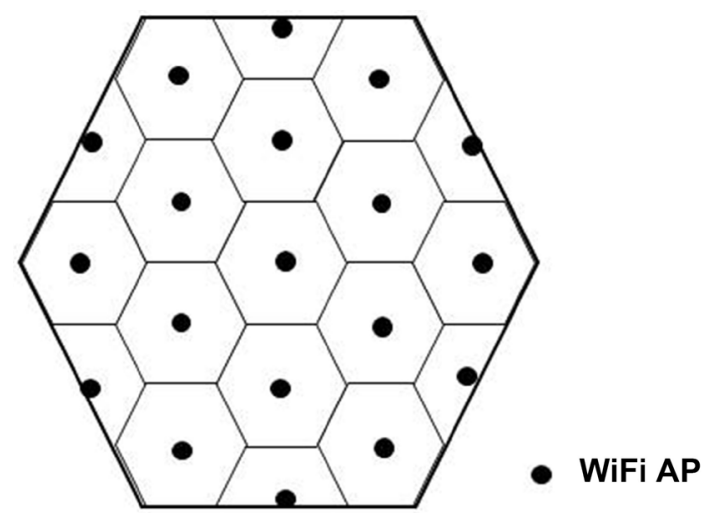

Figure 1. An overlay network with ' $\mathrm{K}$ ' WiFi APs deployment covering a regular hexagonal LTE-A cell.

In our paper, the selection of the LTE transferred users is not random. Instead, it is based on the users with heavy data consumption depending on the requested throughput and transmitted power, so the minimum needed number of WiFi APs is calculated to cope with the traffic of those transferred users as previously described. In addition, the offloading decision is not random or based on the probability of WiFi channels occupation or on the Channel State Information (CSI) either. Instead, it is based on the exact information sent by the $\mathrm{Wi}$ Fi network informing the LTE eNB about its remaining average capacity.

This remaining average capacity depends on the estimated channels load of the physical layer of the WiFi network [9]. In this paper, we consider the average of the channels load or occupation value of the channels that has been calculated in [9], however this value has been averaged for several days during the peak hour traffic of the WiFi network. Based on this averaged value, we have a global estimation calculated through the multiple APs to be collected on a higher control node of the network to estimate the remaining available capacity and to facilitate the measurements collection and processing time.

Therefore, our framework is divided into two phases to transfer cellular data traffic from LTE BS to WiFi:

- The first phase is to determine the heavy users who will transmit the higher power and thus should be offloaded from the LTE system.

- The second phase is WiFi APs dimensioning. This is considered through WiFi APs remaining capacity calculation, and it is based on the remaining throughput of each WiFi AP based on the average occupation or load value of the physical channels.

\section{Problem Formulation}

The WiFi network should assure a minimum acceptable and predefined average per user throughput for an efficient LTE offloading. Based on this average per user throughput, we will calculate the minimum required number of WiFi APs in the overlay network.

As illustrated in Figure 1, we consider a scenario with one LTE BS and $K \mathrm{Wi}$ - 
Fi APs operating separately in licensed and unlicensed spectrum, respectively.

In our scenario, we assume a coverage area of 802.11n WiFi APs with no interference, each transmitting on an orthogonal channel in the $2.5 \mathrm{GHz}$ unlicensed spectrum, selected based on the minimal calculated load value of the channels referring to the algorithm in [9]. This model has also been adopted in other literatures, such as [4] and [7]. Following the same principle, the analysis of $5 \mathrm{GHz}$ spectrum and 802.11ac could be applied [9].

The coexistence of WiFi and LTE could be facilitated by assuming that an inter-system coordinator exists, which performs the WiFi user transfer and resource allocation, as in [7]. To note that our proposed system is very useful for the case where LTE-A and WiFi are deployed by the same network operator, in this case, the inter-system coordinator can be implemented by the cellular network operator itself. Otherwise, it can be implemented by a third-party vendor that provides service enhancement for both WiFi and LTE.

In our paper, the basics of the problem formulation for the LTE eNB are an energy minimization problem and not a throughput maximization problem. The energy minimization solution consists in identifying the users who consume the highest energy and require high throughput rates which are considered in our simulation greater or equal to $20 \mathrm{Mbps}$ [10]. This decision affects the capacity of the WiFi network, as the offloaded users should be in range with an AP having an adequate capacity.

In order to determine the heaviest users in LTE that should be offloaded to WiFi network, the operator needs to determine the resource allocation policy, in terms of Resource Blocks (RBs) assignment and transmission power [8].

We consider the downlink operation of one LTE-A macro cellular BS for a time period of $T$ subframes, possibly expanding over multiple frames. There exists a set of $N_{c}$ users within the cell.

The BS has a set of $M$ available RBs that can be allocated to users in each subframe $(t=1,2, \cdots, T)$. The value of $M$ depends on the available spectrum. Hence, there are in total $(M * T)$ RBs. The system is considered quasi-static, i.e., users do not join or leave the cell during the current time period, and channels do not change significantly (flat fading). Note that, even if channels change rapidly, the eNB will not be aware of this fact, as users transmit their Channel Quality feedback Information (CQI) parameters only once during this time period.

In the beginning of the period, the eNB devises the RB assignment and power allocation policy for serving his users.

Let $x_{n m}(t) \in\{0,1\}$ denote whether RB $m \in M$ is allocated to user $n \in N_{c}$ during subframe $t$.

Let $P_{n m}(t)$ denote the respective transmission power. For each RB, the BS can determine a different transmission power. However, the total power consumption should not exceed a maximum level of aggregated transmission power $P_{\max }$ (Watt). 
Assuming orthogonal allocation of RBs, and ignoring inter-cell interference, i.e., we assume that proper Enhanced Inter-Cell Interference Coordination (eICIC) techniques are applied, the instant rate for each user $n$ is calculated by [8]:

$$
r_{n}(t)=\sum_{m=1}^{M} x_{n m}(t) \cdot W_{b} \cdot \log \left(1+\frac{h_{n m} \cdot x_{n m}(t) \cdot P_{n m}(t)}{\sigma^{2}}\right)
$$

where $W_{b}$ is the symbol rate per $\mathrm{RB}, h_{n m}$ the channel gain of user $n$ in $\mathrm{RB}$ $m$ during the current time period, $\sigma^{2}$ is a parameter considering the variance of the noise [11]. These parameters are estimated through the CQI feedback that is provided by the users, once every period $T$. Based on this policy, the operator determines which users consume the highest power and hence are most costly and should be transferred to WiFi.

\section{WiFi Dimensioning Method}

In this section, the proposed dimensioning method for the minimum needed number of WiFi APs $K$ is presented.

\subsection{Available WiFi Capacity}

To calculate the WiFi network remaining capacity, we need to measure the network load or occupation level.

The channels occupation in WiFi systems may be measured through the standard physical carrier sense mechanism Clear Channel Assessment (CCA), which listens to the received energy on the radio interface.

CCA is defined in the IEEE 802.11-2007 standards as part of the Physical Medium Dependent (PMD) and Physical Layer Convergence Protocol (PLCP) layer. Carrier sense refers to the ability of an AP receiver to detect and decode an incoming WiFi signal preamble. CCA must be reported as Busy when another WiFi signal preamble is detected, and must be held as Busy for the length of the received frame as indicated in the frame's PLCP Length field. Typically, any incoming WiFi frame whose PLCP header can be decoded will cause CCA to report the medium as busy for the time required for the frame transmission to complete [12].

However instead of adopting the instant CCA info on each WiFi AP on the network to reflect the network occupation, we rely in this paper on the channel load estimation method previously analyzed in [9], which enables to scan and measure the occupation of all WiFi overlapped physical channels simultaneously, collected on a higher control node, instead of the local measurement on each AP. This load estimation method facilitates the occupation measurements aggregation and processing time.

In addition, since initially this value is an instant occupation measure, we consider in this paper the average value of channels occupation during peak hours for several days within the LTE-WiFi HetNet, so the dimensioning calculations will be based on an averaged occupation value for several days to reflect more accurately the load of the WiFi network. 
Let $\alpha$ denotes the average load or occupation value of channel $i ;\left(1-\alpha_{i}\right)$ is therefore the available idle capacity of this WiFi channel.

In addition, since WiFi APs operate on the different 12 channels of the 802.11n system based on the minimum load value of the channel [9], different APs might be operating simultaneously on a specific channel $i$, taking into consideration that they are not neighbor APs to avoid the inter-channel interference. Therefore, the total available capacity of this channel $i$ will be divided between at least two APs. If we consider $t_{i}$ as the number of APs operating simultaneously under the different frequencies of the WiFi channels $\left(1<t_{i}<12\right)$, we can deduce the below equation [13]:

$$
K=\sum_{i=1}^{12} t_{i}
$$

$K \geq 1$ is the number of WiFi APs to be calculated.

Consequently, we can define the available capacity in terms of bit rate for a WiFi AP $l(l=1, \cdots, K)$, measured on a frequency of channel $i$, and the capacity for the whole WiFi network, denoted as $R_{l}^{i}$ and $R_{t o t}$ respectively, as follows:

$$
\begin{gathered}
R_{l}^{i}=R_{w_{\max }} \cdot \frac{\left(1-\alpha_{i}\right)}{t_{i}} \\
R_{t o t}=\sum_{i=1}^{K} \sum_{i=1}^{12} \mu_{l}^{i} \cdot R_{l}^{i}
\end{gathered}
$$

where $R_{w_{\max }}$ is the maximum throughput of the WiFi APs (considered as same releases and specs), $i$ is WiFi channel number $(i=1, \cdots, 12), R_{t o t}$ is the total remaining capacity or throughput of the WiFi network, $R_{l}^{i}$ is the remaining capacity or throughput of the WiFi AP $l(l=1, \cdots, K)$ measured on frequency $i$, and $\mu_{l}^{i}=0,1$ is 1 if the $\mathrm{AP} l$ is operating on frequency $i$, and 0 if the AP $l$ is not operating on frequency $i$.

From Equation (4), we can estimate the total available capacity of the WiFi network, and thus dimension the minimum needed number of WiFi APs that will handle the transferred LTE users according to certain throughput criteria that will be analyzed in the next section.

\subsection{Dimensioning of the WiFi Network}

To ensure the same user experience, the average per-user throughput offered by the WiFi network should be at least equal to or higher than the cellular network throughput.

Based on this constraint, we set the target average per-user WiFi throughput as follows [4]:

$$
S_{W}^{\text {user }} \geq S_{C}^{\text {user }}
$$

where $S_{W}^{u s e r}$ and $S_{C}^{u s e r}$ represent the average per-user WiFi throughput and the average per-user cellular throughput respectively.

We define a maximum throughput threshold within the LTE network, considered in the simulations as 20 Mbps as average [10], where each user exceeding 
this threshold is considered as heavy user and should be transferred from LTE to WiFi. From Equation (4) we can conclude the below equation:

$$
\operatorname{Avg}\left(S_{W}^{u s e r}\right)=\frac{R_{t o t}}{N_{w}}
$$

where $N_{w}$ is the number of the heavy users to be transferred from LTE to WiFi as previously described.

While setting the maximum throughput threshold within the LTE as the minimum needed throughput per user to be ensured by the WiFi network, we calculate the minimum required number of WiFi APs $K$ that achieves the target average per user WiFi throughput.

We can express the mathematical expression of $K$ by:

$$
K=\underset{K}{\operatorname{argmin}} S_{W}^{\text {user }}
$$

where $\operatorname{Avg}\left(S_{W}^{u s e r}\right) \geq r_{n}(t), r_{n}(t)$ is calculated in Equation (1).

\section{Profit Estimation}

LTE and WiFi operators seek a monetary profit in case of cooperation while heavy users are transferred from LTE to WiFi. Each player WiFi or LTE tries to adopt a network configuration that decreases its own costs in order to maximize its profits. Thus, we evaluate in this section the Shapley Value that proved to be very effective in profit sharing in a multiplayer context, where several types of relationships are involved [14]. The idea is that each player will have a profit share proportional to its contribution in the network setting and the added value it brings to the overall value chain.

\subsection{The Shapley Value: Definition and Properties}

The Shapley value is the share gained by a player $i$ when he is in coalition $S$. This value $\varphi_{i}(S, V)$ as defined by Shapley in [14] and [15] is given by:

$$
\varphi_{i}(S, V)=\frac{1}{N !} \sum_{\pi \in \Pi} \Delta_{i}(V, S(\pi, i)) \forall i \in N
$$

where $N$ is the set of players and $S$ a given coalition formed by a subset of these players, $V(S)$ is the worth function that denotes the weight or payoff of coalition $S, \Pi$ is the set of all $N$ ! players permutations, $S(\pi, i)$ is the coalition formed by players from rank 1 till $i$ in a given permutation. $\pi \in \Pi$ and $\Delta_{i}(V, S(\pi, i))=V(S)-V(S \backslash\{i\})$ is the marginal contribution of player $i$ in coalition $S$ defined as the difference between the worth functions of $(S)$ and $(S \backslash\{i\})$ and representing the benefits or losses that player $i$ could bring if he entered coalition $(S \backslash\{i\})$.

Note that the Shapley value has the following additivity property: if the worth function $V(S)$ can be divided into two components $V(S)=V_{1}(S)+V_{2}(S)$, then the Shapley value is equal to:

$$
\varphi_{i}(S, V)=\varphi_{i}\left(S, V_{1}\right)+\varphi_{i}\left(S, V_{2}\right)
$$




\subsection{Profit Sharing Using Shapley Value}

In our model, there are two players only, LTE and WiFi, considered managed by the same operator in Scenario 1, and by different operators in Scenario 2.

The profit is the difference between the total revenue and costs, and is to be shared among the different players in the system. Using the above defined Shapley additivity property, the worth function of any coalition $S$, i.e., its payoff $V(S)$, is simply the difference of the revenue worth function $V_{r}(S)$ andthe cost worth function $V_{c}(S)$. This yield the profit share of each player $i$ as follows [14]:

$$
\varphi_{i}(S, V)=\varphi_{i}^{r}\left(S, V_{r}\right)-\varphi_{i}^{c}\left(S, V_{c}\right)
$$

where $r$ and $c$ are the revenue and cost components respectively.

We now derive closed-form expressions for the Shapley value so as to ease its numerical computation and overcome the exhaustive summation in Equation (8).

\subsubsection{Revenue Sharing}

Revenue depends on the pricing of data traffic offered to mobile users, and the volume of this traffic. In general, operators offer various data bundles with a flat rate for each one. Therefore, by having the total number of mobile subscribers within the LTE network, $N_{L}$, and the number of users transferred to the WiFi network, $N_{W}$, along with their related average Mbps volume per month, the operator can estimate the related revenues.

Let $\gamma_{L}$ and $\gamma_{w}$ be the total average volume in Mbps per month per user connected on LTE and per user transferred to WiFi respectively. This volume is calculated based on an average value per month calculated from Equation (1). $\lambda$ is the price per Mbps per user in LTE network as presented in Table 1.

Table 1. System configuration.

\begin{tabular}{cc}
\hline Parameters & Values \\
\hline Bandwidth & $10 \mathrm{MHz}$ \\
Duration & $10 \mathrm{~ms}$ \\
RBs per Time Slot & 50 \\
RBs per TTI & 100 \\
Subcarriers per RB & 12 \\
Max eNB TX Power & $43 \mathrm{dBm}$ \\
Max UE TX Power & $23 \mathrm{dBm}$ \\
Symbols per RB & 7 \\
Number of subframes (T) & 20 \\
Block Error Rate & 0.1 \\
Channel Gain & $6 \mathrm{~dB}$ \\
Max WiFi AP & $600 \mathrm{Mbps}$ \\
Cost of LTE BS & $45,000 \mathrm{USD}$ \\
Cost of WiFi AP & $500 \mathrm{USD}$ \\
Cost 1 Mbps Data Traffic & $0.001 \mathrm{USD}$ \\
\hline &
\end{tabular}


The revenues of the network in presence of LTE only $\left(G_{L}\right)$, and in presence of LTE and WiFi $\left(G_{L, W}\right)$ are calculated as per the below equations respectively:

Case where WiFi supports LTE:

$$
\begin{gathered}
G_{L}=\left(N_{L}-N_{W}\right) * \gamma_{L} * \lambda \\
G_{L, W}=\left(N_{L}-N_{w}\right) * \gamma_{L} * \lambda+N_{w} * \gamma_{w} * \lambda
\end{gathered}
$$

Case where WiFi does not support LTE:

$$
\begin{gathered}
G_{L}=N_{L} * \gamma_{L} * \lambda \\
G_{L, W}=N_{L} * \gamma_{L} * \lambda
\end{gathered}
$$

Indeed, in the scenario where WiFi does not support LTE, WiFi will not be participating in the users offload or the revenues sharing, therefore the total LTE users $N_{L}$ will stay connected to the LTE network, and therefore $G_{L}$ and $G_{L, W}$ are equal.

Revenues in presence of WiFi network only are not applicable, since this case is not considered, thus $G_{w}=0$.

By applying the Shapley value of Equation (8), we calculate the share of both LTE and WiFi in the revenues, assuming the different permutation of the two players (LTE-WiFi, then WiFi-LTE) as per the below equations:

$$
\begin{gathered}
\varphi_{L}^{r}=\frac{1}{2} \cdot\left(G_{L}+G_{L, W}\right) \\
\varphi_{W}^{r}=\frac{1}{2} \cdot\left(G_{L, W}-G_{L}\right)
\end{gathered}
$$

$\varphi_{L}^{r}$ and $\varphi_{W}^{r}$ are the shares in revenues of LTE and WiFi respectively.

\subsubsection{Cost Sharing}

The cost of equipment and related operations expenditure for the LTE BS and WiFi $\mathrm{AP}$ are $\mathrm{C}_{\mathrm{LBS}}$ and $\mathrm{C}_{\mathrm{WAP}}$ respectively presented in Table 1.

In addition, based on Equation (8), the cost shares of the network in presence of LTE only $\left(C_{L}\right)$, and in presence of LTE and WiFi $\left(C_{L, W}\right)$ are calculated as per the below equations:

$$
\begin{gathered}
C_{L}=L \cdot C_{L B S} \\
C_{L, W}=\left(K \cdot C_{W A P}\right)+\left(L \cdot C_{L B S}\right)
\end{gathered}
$$

$K$ is the number of WiFi APs calculated in Equation (7), and $L$ is the number of LTE BSs that will assure an average throughput per user greater than $20 \mathrm{Mbps}$ for around 100 simultaneous active users [10] (minimum values for $L$ are considered as follow: $L=1$ in case of WiFi support, $L=2$ in case WiFi does not support LTE).

Similarly, the cost in presence of WiFi network only is not applicable since this case is not considered, thus $C_{W}=0$.

The same method based on Shapley value is applied for the cost shares of LTE and WiFi to get the below equations: 


$$
\begin{gathered}
\varphi_{L}^{c}=\frac{1}{2} \cdot\left(C_{L}+C_{L, W}\right) \\
\varphi_{W}^{c}=\frac{1}{2} \cdot\left(C_{L, W}-C_{L}\right)
\end{gathered}
$$

\subsubsection{Profit Sharing}

The profit distribution of each player is simply the difference between its revenue and cost share as per Equation (10).

We consider as previously described two scenarios:

- Scenario 1: the case of a single, joint LTE/WiFi operator, where the same operator owns both LTE and WiFi infrastructures.

- Scenario 2: the case where the LTE and WiFi are owned by separate operators.

For both scenarios, we calculate the profit in case WiFi APs support LTE for its heavy users and in case there is no WiFi support.

In Scenario 1, we consider the total cost share, revenue share and profit share as per the below equations:

$$
\begin{gathered}
\varphi^{c}=\varphi_{L}^{c}+\varphi_{W}^{c} \\
\varphi^{r}=\varphi_{L}^{r}+\varphi_{W}^{r} \\
\varphi=\varphi^{r}-\varphi^{c}
\end{gathered}
$$

whereas in Scenario 2, the profit share is calculated separately for LTE and WiFi as per the below equations:

$$
\begin{gathered}
\varphi_{L}=\varphi_{L}^{r}-\varphi_{L}^{c} \\
\varphi_{W}=\varphi_{W}^{r}-\varphi_{W}^{c}
\end{gathered}
$$

Due to its fairness, the profit distribution under Shapley value is appealing in cooperative games. Each player is rewarded a profit proportional to its contribution to the overall profit. This is demonstrated in the simulation results section, where the profit started to be positive or beneficiary, in case of WiFi support, earlier than the case of without WiFi support. This is due to the fact that the cost of investment in WiFi is much less than the additional cost of investment for the LTE BSs, with same subscribers' revenues and offered throughput per user.

\section{Simulation Results and performance Evaluation}

We consider in our simulations, an LTE FDD system for one eNB cell operating in $1800 \mathrm{MHz}$ with an available bandwidth of $10 \mathrm{MHz}$ [8] [16]. The WiFi network is based on $802.11 \mathrm{n}$ system that operates in $2.5 \mathrm{GHz}$ bandwidth with 12 overlapped channels on the physical layer [9]. Every Transmission Time Interval (TTI), the eNB makes a scheduling decision to dynamically assign the available time-frequency RBs to the UEs. The eNB scheduler aims at power minimization while also at satisfying UEs demands.

Table 1 summarizes the basic system model configuration, while considering a total number $N_{c}$ of LTE users operating in the heterogeneous network vary- 
ing from 10 to 100 users per eNB making simultaneously data sessions.

Based on the configured setup, we present in this section, numerical results by using MATLAB to analyze the minimum required number of WiFi APs versus LTE and WiFi throughput.

By varying the number of simultaneous active users in the LTE cell from 10 to 100 active users, Figure 2 represents the number of users considered as heavy users and that need to be offloaded to WiFi network.

Taking into consideration that the LTE users will be offloaded when their demands exceed the $20 \mathrm{Mbps}$, considered as the average per user throughput in LTE-A network [10], the minimum needed number of WiFi APs, and the acquired throughput in the WiFi network are shown in Figure 3 and Figure 4 respectively, noting that there is no restriction in this case on the maximum offered throughput per user in the WiFi network.

As we can observe, with few LTE users to be offloaded, the WiFi network with only one AP can provide up to around $120 \mathrm{Mbps}$ as theoretical value on top of its existing users. The WiFi throughput per user decreases with the additional number of offloaded simultaneous active users, with an average of $40 \mathrm{Mbps}$, thus greater than the maximum defined threshold in the LTE network (20 Mbps).

By adopting this method, in addition to the saved cost when increasing the WiFi APs in indoors environment, to a maximum of 4 APs as shown in Figure 3 , instead of increasing the number of eNBs; the user experience will be enhanced instead of suffering from any possible congestion or throughput deterioration with limited number of LTE eNBs.

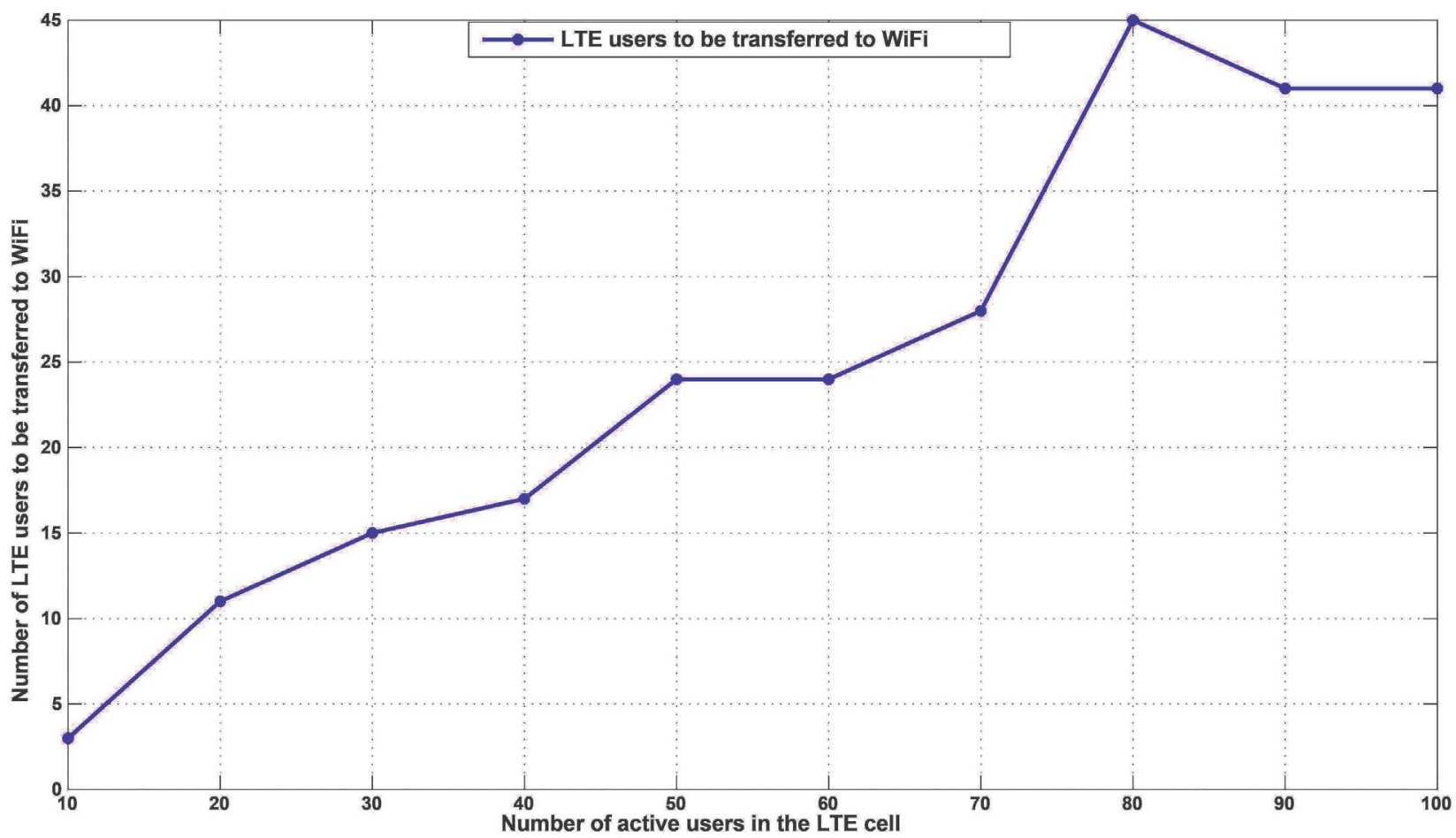

Figure 2. Number of users to be offloaded to WiFi with respect to the total number of active users in the LTE cell. 


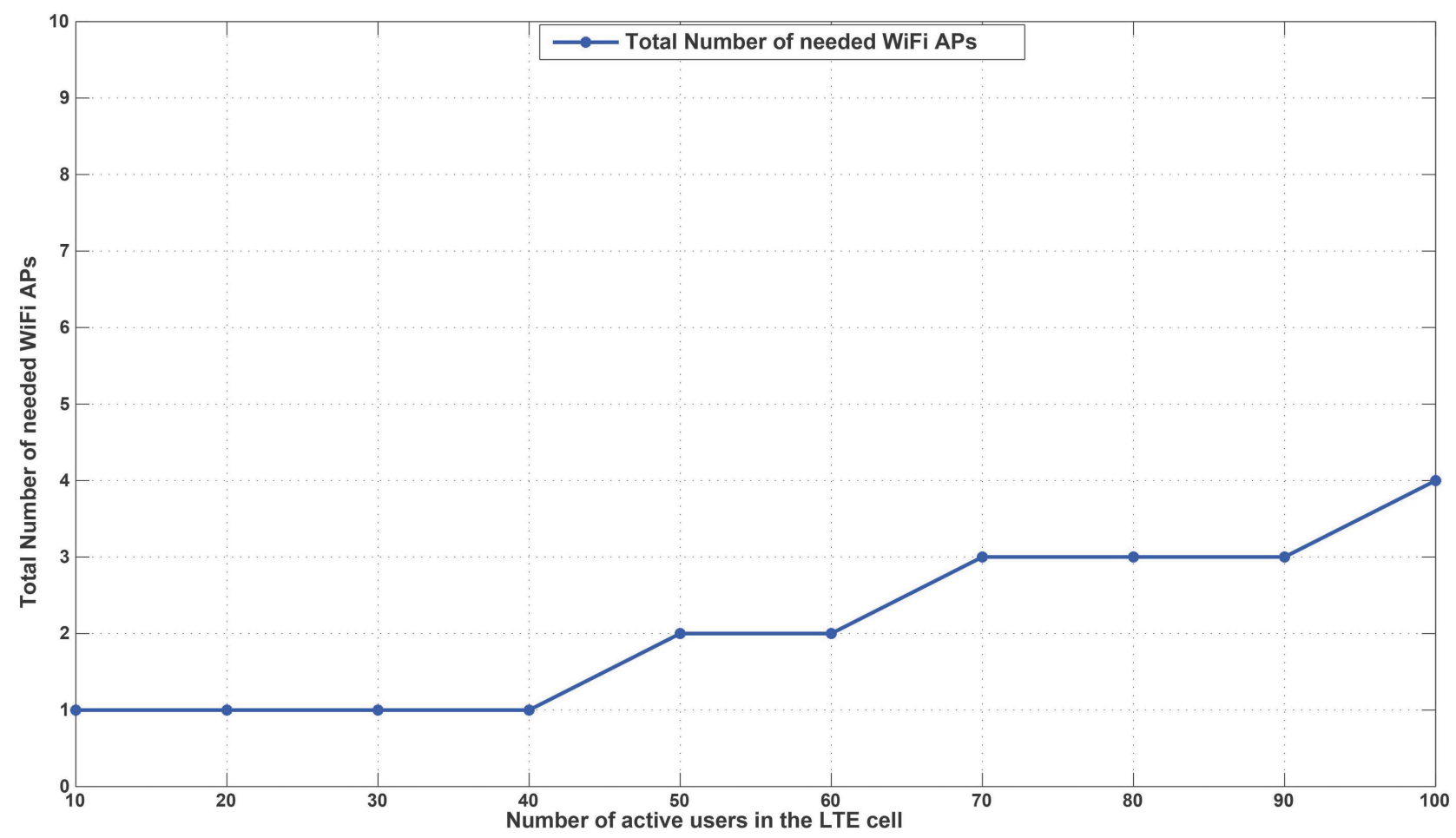

Figure 3. Total number of needed WiFi APs with no limitation on average per user WiFi throughput.

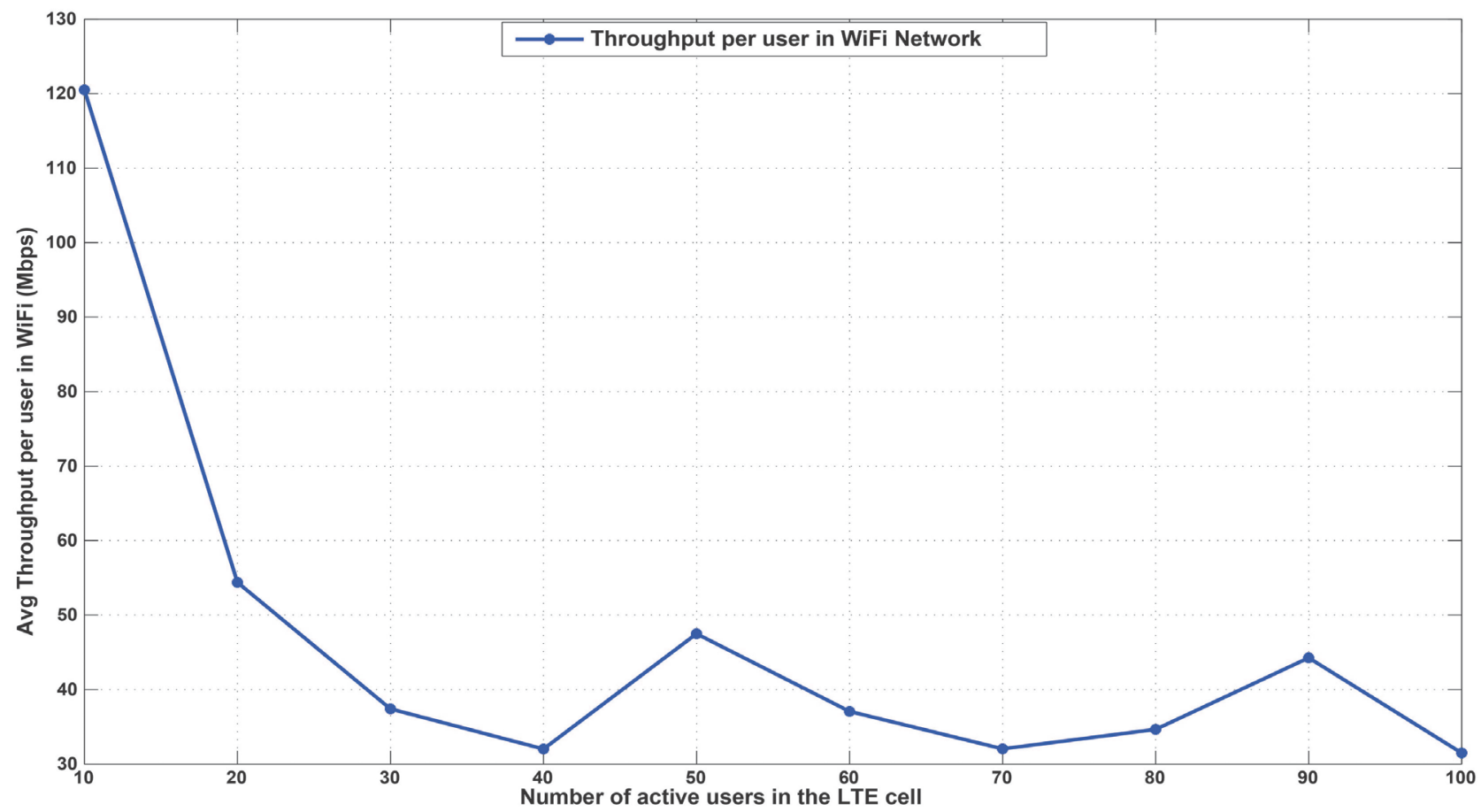

Figure 4. Average per user WiFi throughput (Mbps).

If we take the scenario of a restricted threshold of throughput offered to the offloaded users in the WiFi network (e.g. a max of $20 \mathrm{Mbps}$ ), the needed number of WiFi APs will be reduced to 3 APs as presented in Figure 5. 


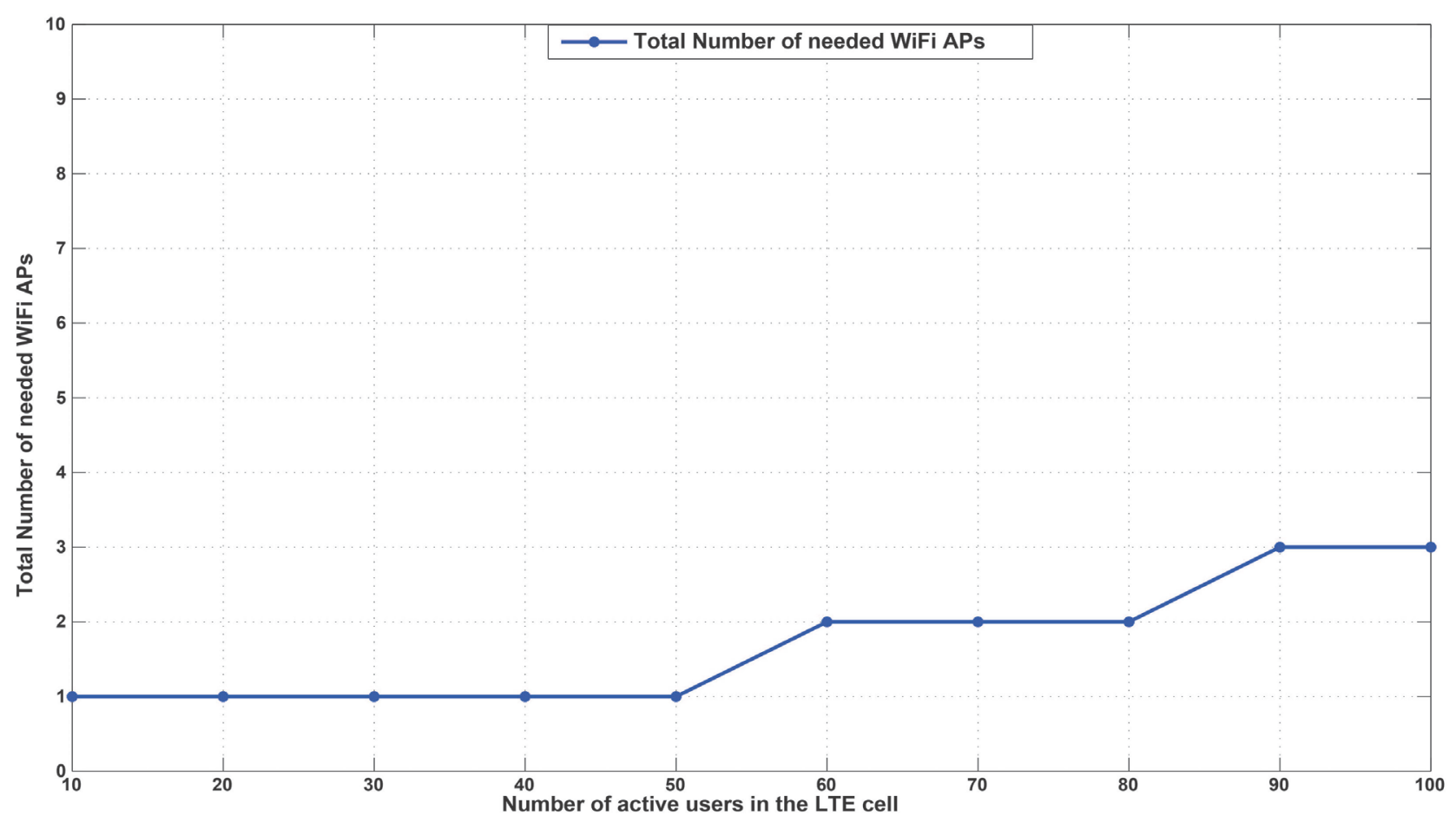

Figure 5. Total number of needed WiFi APs with average per user WiFi throughput set to $20 \mathrm{Mbps}$ maximum.

To pinpoint the saving in LTE when applying our proposed dimensioning method, we have measured the average power consumption saving related to the transmitted power after being transferred to WiFi.

Figure 6 and Figure 7 represent the average saved power consumption in the eNB in Watts, and the percentage of power saving in respect to the total consumed power, respectively. As we can observe, there is on average $40 \%$ saving of the total consumed power in the eNB. This saving is expected to grow obviously when the number of offloaded users increases. As we can observe the average power consumption in Figure 6 is proportional to the number of users to be offloaded to WiFi in Figure 2 since this power consumption is the total capacity in Watt consumed by the offloaded users.

Finally, the results of the profit calculations based on Shapley value are presented in Figure 8 and Figure 9 for Scenario 1 (joint operator) and Scenario 2 (separate operators), respectively.

The study is spread over 10 months where we only consider that the subscribers' number is constant during this period without additional growth on LTE network (data traffic and network expansion). In this case, we consider that a minimum of $1 \mathrm{BS}$ is needed for 100 simultaneous active users with WiFi network support, and 2 BSs are needed in case of no WiFi support. In Figure 9, we notice that after 10 months, the profit of LTE without WiFi support could be the same as if there is WiFi support. We should note that, after this period, the taken assumptions of the growth of subscribers and consequently the growth of revenues and the needed number of WiFi APs will be changed, however this analysis is not considered in our paper at this stage. 


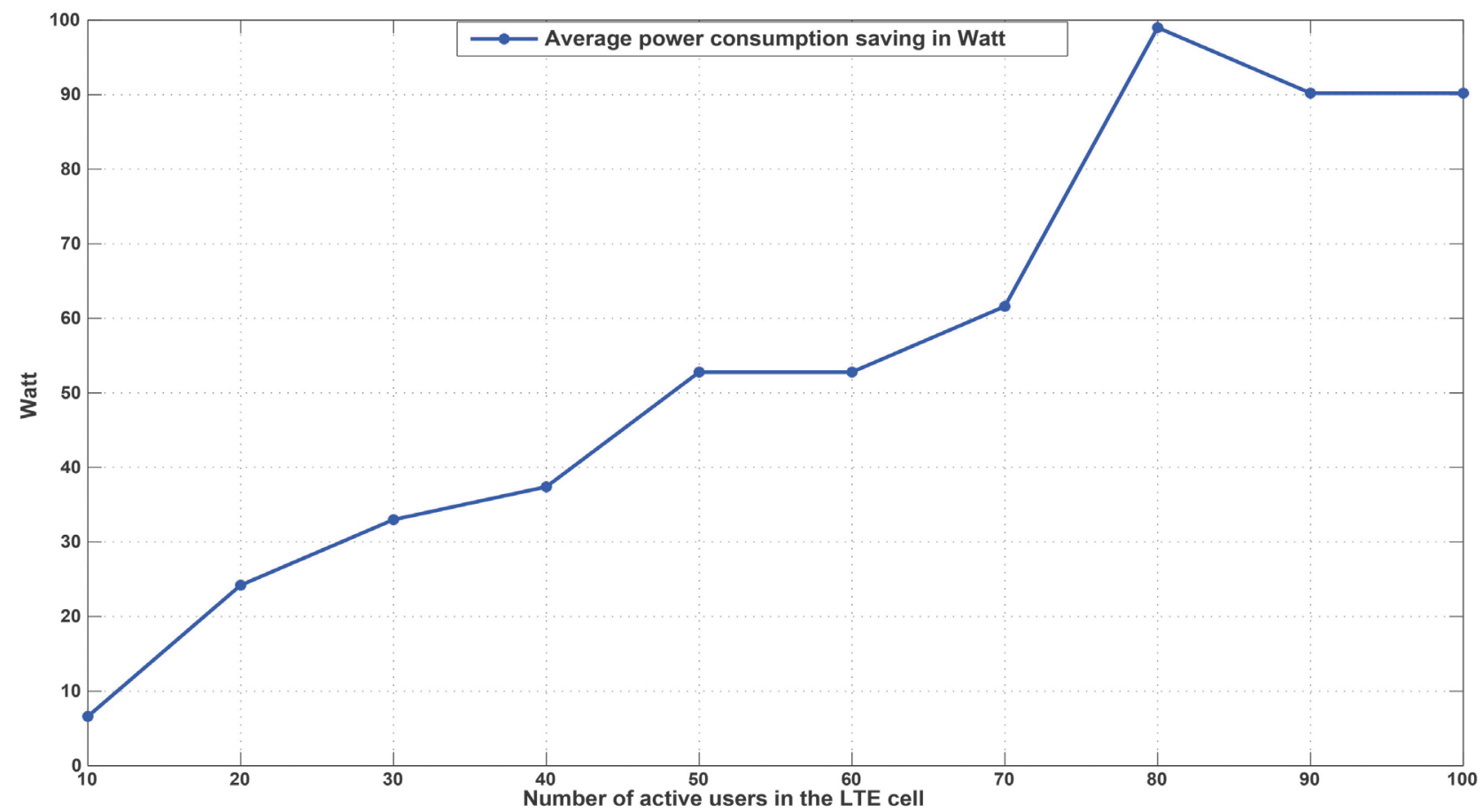

Figure 6. Average power consumption saving in Watt.

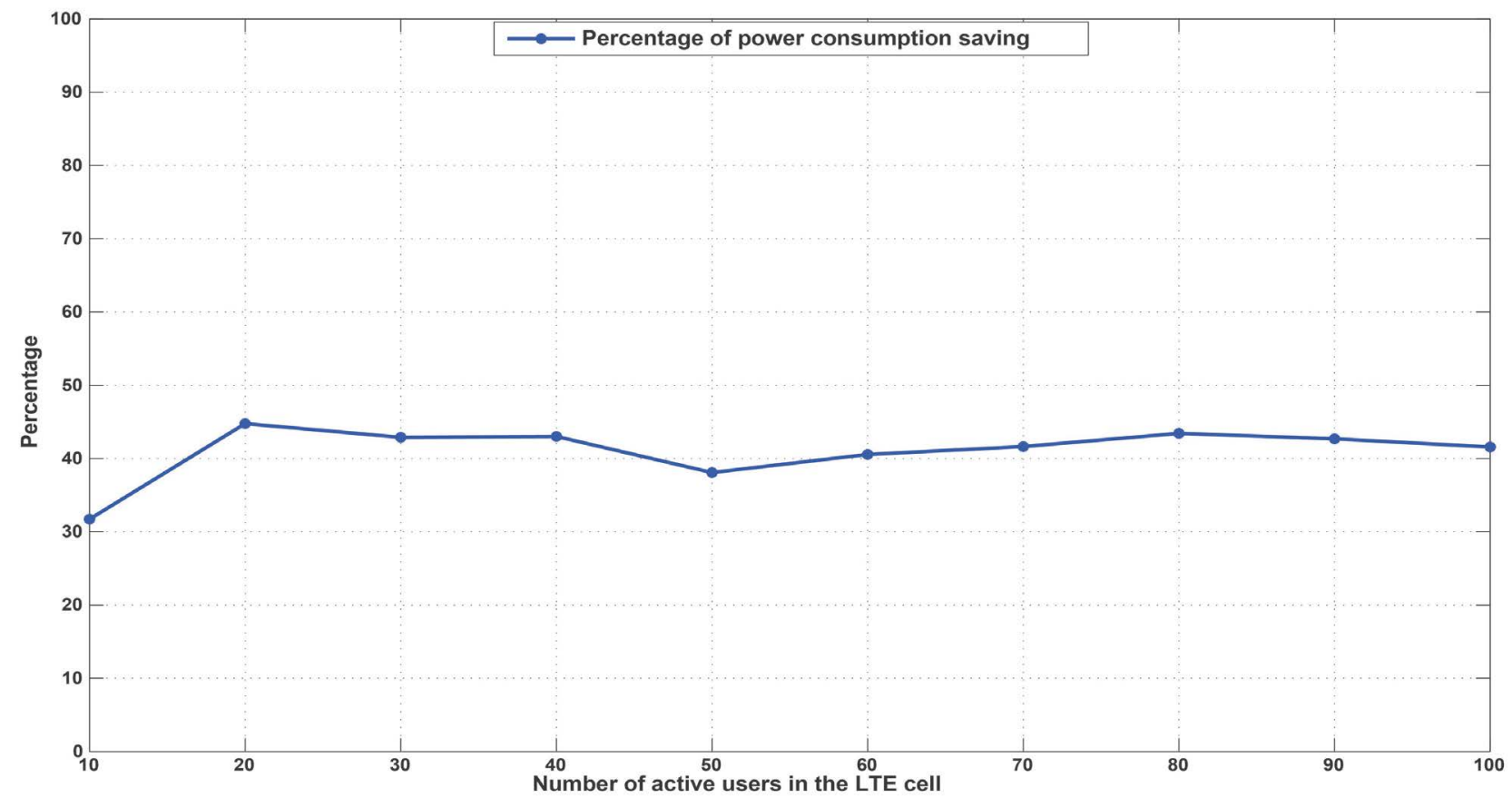

Figure 7. Percentage of power consumption saving.

In case of joint operator in Figure 8, we can observe that the breakeven point for the profit is found almost starting the 5th month where the revenues share become higher than the investment cost in case WiFi supports LTE. However, this gain is much more delayed for almost several additional months in case there is no WiFi support. 


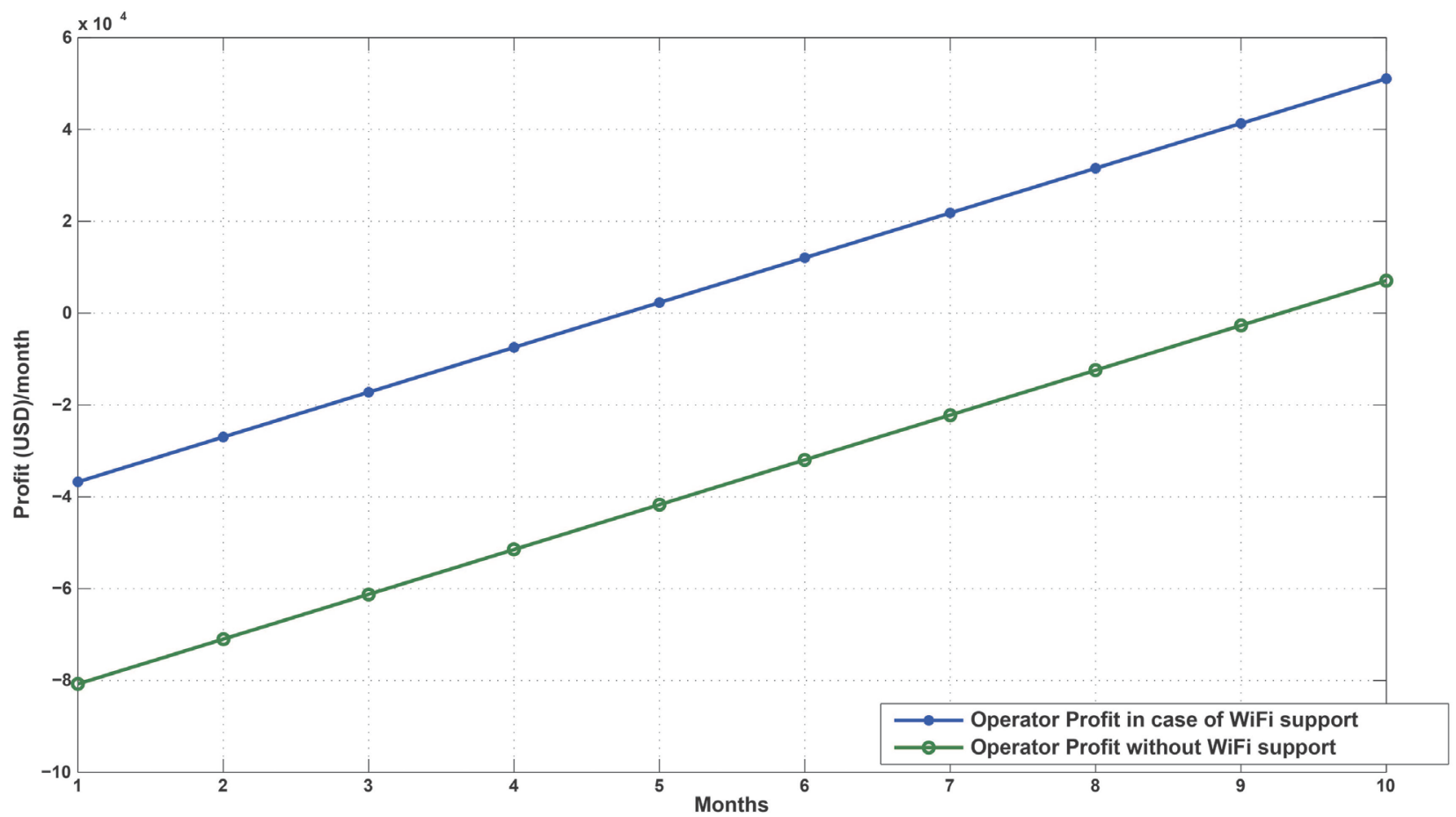

Figure 8. Profit in case of joint WiFi/LTE operator.

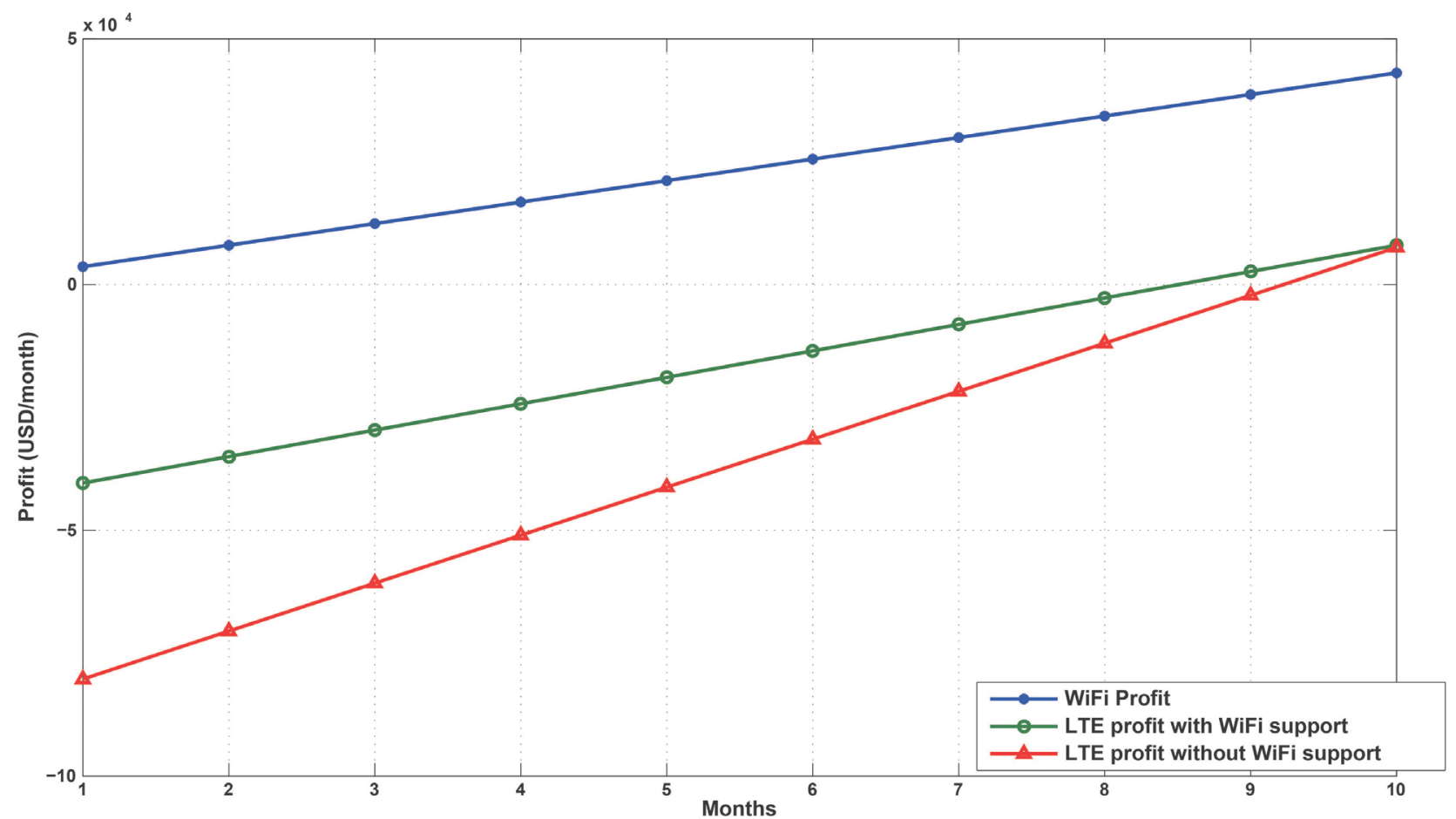

Figure 9. Profit in case of separate WiFi and LTE operators.

In case of separate operators in Figure 9, the positive profit for the LTE is noticeable starting the 9th month, whereas it remains less than the profit in case of joint operator with WiFi support. 
In addition, we can observe that the profit of the WiFi is always positive in case of separate operators as shown in Figure 9, since the traffic transferred to $\mathrm{WiFi}$ is directly covering the investment expenses or cost.

Finally, we can conclude that the Return on Investment (ROI) is maximum in the scenario where the operator owns both WiFi and LTE networks, and while WiFi is providing support to LTE.

\section{Conclusions}

In this paper, we have proposed a mathematical approach to find the minimum required number of WiFi APs to support the heavy users' traffic transferred from LTE to WiFi network, based on the remaining available capacity of the WiFi network.

This capacity was estimated considering the overlapping characteristics of the physical channels of the WiFi technology, where we can estimate the average percentage of busy time and idle time of the channels during peak hours traffic and for several days to estimate the global occupation and thus capacity of the WiFi network.

The benefit of the proposed WiFi dimensioning method, which cooperates with LTE to handle the heavy users' traffic, is presented through the profit calculations by applying the gaming theory Shapley Value. The estimated profit using Shapley value is maximal when the same operator owns both WiFi and LTE networks and while WiFi is supporting LTE.

Furthermore, through the mathematical approach proposed in our paper, we can ensure an efficient coexistence between LTE-A and WiFiHetNets, while providing a high level of bit rate to the end users, and with minimum required hardware and investment cost.

Further studies could be performed to include the transfer of users from WiFi to LTE to ensure both directions cooperation, and the transfer of LTE users to WiFi based on the coverage area and distance between the APs and the transferred users. In addition, we can consider the performance degradation due to hidden and exposed node problems and investigate the impact of practical traffic patterns and multiple modulation and coding scheme (MCS) levels on WiFi cell deployment. Finally, with the deployment of Internet of Things, uplink traffic will drastically increase. Therefore, uplink resource allocation in mobile networks will become also a crucial problem to solve and analyze in future studies.

\section{Conflicts of Interest}

The authors declare no conflicts of interest regarding the publication of this paper.

\section{References}

[1] Sun, H.Y., et al. (2017) Enabling LTE and WiFi Coexisting in $5 \mathrm{GHz}$ for Efficient Spectrum Utilization. Journal of Computer Networks and Communications, 2017, 
Article ID: 5156164. https://doi.org/10.1155/2017/5156164

[2] Elgendi, I., et al. (2017) Traffic Offloading for 5G: L-LTE or Wi-Fi. IEEE Conference on Computer Communications Workshops (INFOCOM WKSHPS): IECCO: Integrating Edge Computing, Caching, and Offloading in Next Generation Networks, Atlanta, 1-4 May 2017. https://doi.org/10.1109/INFCOMW.2017.8116470

[3] Cheng, R.-S., et al. (2018) WiFi Offloading Using the Device-to-Device (D2D) Communication Paradigm Based on the Software Defined Network (SDN) Architecture. Journal of Network and Computer Applications, 112, 18-28.

[4] Kim, J.Y., et al. (2013) Placement of WiFi Access Points for Efficient WiFi Offloading in an Overlay Network. IEEE 24th International Symposium on Personal, Indoor and Mobile Radio Communications: Mobile and Wireless Networks, London, 8-11 September 2013. https://doi.org/10.1109/PIMRC.2013.6666673

[5] Wang, T., et al. (2010) Efficient WiFi Deployment Algorithms Based on Realistic Mobility Characteristics. IEEE MASS, San Francisco, 8-12 November 2010. https://doi.org/10.1109/MASS.2010.5663941

[6] Dimatteo, S., et al. (2011) Cellular Traffic Offloading through WiFi Networks. IEEE MASS, Valencia, 17-22 October 2011. https://doi.org/10.1109/MASS.2011.26

[7] Chen, Q.M., et al. (2016) Rethinking Mobile Data Offloading for LTE in Unlicensed Spectrum. IEEE Transactions on Wireless Communications, 15, 4987-5000. https://doi.org/10.1109/TWC.2016.2550038

[8] Apostolaras, A., et al. (2014) C2M: Mobile Data Offloading to Mesh Networks. IEEE Global Communications Conference, Austin, 8-12 December 2014. https://doi.org/10.1109/GLOCOM.2014.7037578

[9] Saliba, D., et al. (2017) Overlapped Physical Channels Load Measurement in 802.11 Networks. International Journal of Advanced Research in Computer Science, 8. https://doi.org/10.26483/ijarcs.v8i8.4810

[10] Singh, K.P. and Chopra, P.K. (2014) Throughput Computation of LTE-A Network for Urban Area. International Journal of Advanced Research in Electronics and Communication Engineering, 3.

[11] Wang, Y.P., et al. (2012) Self-Optimization of Downlink Transmission Power in 3GPP LTE-A Heterogeneous Network. Vehicular Technology Conference, Quebec City, 3-6 September 2012. https://doi.org/10.1109/VTCFall.2012.6398913

[12] Tanenbaum, A.S. and Wetherall, D.J. (2010) Computer Networks. Fifth Edition.

[13] Saliba, D., et al. (2019) WiFi Dimensioning to Offload LTE in 5G Networks. IEEE Computing and Communication Workshop and Conference, LAS Vegas USA, January 2019.

[14] Razzac, A.A., et al. (2013) Dimensining and Profit Sharing in Hybrid LTE/DVB Systems to Offer Mobile TV Services. IEEE Transactions on Wireless Communications, 12, 6314-6327. https://doi.org/10.1109/TWC.2013.110813.130397

[15] Shapley, L. (1953) Contributions to the Theory of Games II. Annals of Mathematics Studies Vol. 298, Princeton University Press, Princeton, Ch. A Value for n-Person Games, 307-317. https://doi.org/10.1515/9781400881970-018

[16] Bertrand, P. (2011) Channel Gain Estimation from Sounding Reference Signal in LTE. IEEE 73rd Vehicular Technology Conference, Yokohama, 15-18 May 2011. https://doi.org/10.1109/VETECS.2011.5956571 